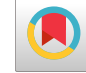

\title{
Application of Modified Flower Petal in Side Branch of Acute Myocardial Infarction Patient: REVERSE Modified Flower Petal
}

\author{
Habib Haybar (iD ${ }^{1}$ and Zeinab Deris Zayeri (iD ${ }^{2, *}$ \\ ${ }^{1}$ Atherosclerosis Research Center, Ahvaz Jundishapur University of Medical Sciences, Ahvaz, Iran \\ ${ }^{2}$ Golestan Hospital Clinical Research Development Unit, Ahvaz Jundishapur University of Medical Sciences, Ahvaz, Iran \\ "Corresponding author: Golestan Hospital Clinical Research Development Unit, Ahvaz Jundishapur University of Medical Sciences, Ahvaz, Iran. Email: \\ zeynabderisgenetice@gmail.com
}

Received 2020 June 27; Revised 2020 July 27; Accepted 2020 September 16.

\begin{abstract}
Introduction: Concerning the poor clinical outcomes in cases of acute myocardial infarction(AMI) with bifurcation lesions undergoing primary percutaneous coronary intervention (PCI), we described a successful application of modified flower petal technique and called it the REVERSE modified flower petal technique in a patient with AMI.

Case Presentation: A 56-years-old man who suffered from chest pain was admitted to the emergency ward. After clinical examination, the primary diagnosis was acute anterolateral MI. Therefore, PCI on left anterior dimension (LAD) and diagonal arteries using modified flower petal stenting was performed in the side branch, which we called it REVERSE modified flower petal.

Conclusions: Modified flower petal stenting was performed by the REVERSE mechanism as described in the report of the new intervention $(2.5 \times 18)$. The follow-up after 24 months by the exercise stress test using the Bruce protocol supported the success of the intervention.
\end{abstract}

Keywords: Myocardial Infarction, Percutaneous Coronary Intervention, Coronary Angiography, Modified Flower Petal

\section{Introduction}

During recent years, the early diagnosis, effective treatment, and suitable management of complications have decreased the rate of morbidity in AMI (1). Although prescribing anti-thrombolytic drugs, particularly at the first hours after AMI, improves blood flow in the occulted artery in $60 \%$ of the patients, the complete restoration of blood flow in the involved artery needs interventional therapy $(2,3)$. There are various two-stent techniques, such as the culotte technique, T-stenting, crush technique, simultaneous kissing stenting. The angulation of the significant side branch (SB) affects the decision on the technique to be chosen. The $\mathrm{SB}$, the main vessel (MV), and the lesion morphology are three important parameters in choosing the technique (4).

In this case, we used a new technique which we called REVERSE modified flower petal stenting during PPCI for a 56-year-old patient who suffered from AMI to increase the chance of rescue of the involved coronary artery and decrease the risk of restenosis. We followed the patient for 19 months by angiography.

\section{Case Presentation}

A 56-year-old man who suffered from typical chest pain, nausea, and diaphoresis, but with no report of dyspnea, vomiting, paroxysmal nocturnal dyspnea (PND), and orthopnea, was admitted to the emergency ward. An initial electrocardiogram (ECG) was performed that showed ST elevation in I and aVL, and ST depression in V2-V6. The patient was diagnosed with Medina type 1.1.1 coronary bifurcation lesions based on the evidence. Transthoracic echocardiogram (TTE) showed mild mitral regurgitation and left ventricular ejection fraction (LVEF) of $\sim 50 \%$ with lateral wall hypokinesis. However, mitral stenosis (MS), aortic regurgitation (AR), and aortic stenosis (AS) were not seen.

\subsection{The Report of the New Intervention We Used for This Emer- gency Case}

To clarify the new intervention, we explain it with a short discussion on flower petal stenting. The procedure starts with wiring main and side branches. It is necessary to pre-dilate all significantly stenosed branches. After the pre-dilation, the SB stent-MV balloon system is prepared 
outside of the guiding catheter. First, the plastic stent cover is pulled back slightly to expose the final proximal stent strut; the stent delivery system balloon is inflated to 5 to $6 \mathrm{~atm}$ and then deflated to expand the final proximal stent strut. The MV wire is then passed through the now expanded, final proximal stent strut (See figure $1 \mathrm{~A}-\mathrm{C}$ in reference (5)).

The stent will be placed in the main branch, and a strut will be placed in the side branch. In this case, the interventionist doctor, Habib Haybar, acted the REVERSE of this intervention. He put the main stent in the side branch; that is why he called this intervention REVERSE modified flower petal stenting (because the side branch was wide and plugged). Then, he put a strut in the main branch (Figure 1).

In a previous study by Cayli et al. (5) entitled "modified flower petal technique: A new technique for the treatment of Medina type 1.1.1 coronary bifurcation lesions" published in the Journal of the American College of Cardiology (JACC), one of the exclusion criteria was patients with acute myocardial infarction, while we used the REVERS of this intervention for a patient with acute myocardial infarction.

The primary diagnosis was acute anterolateral MI and thus, ASA 325 and Plavix ( $45 \mathrm{mg} / \mathrm{kg}$ ) were prescribed for one year, which continued with ASA 325 for the rest of his life. Finally, the patient was transferred to the Catheterization Lab, and pPCI on LAD diagonal using modified flower petal stenting by Biotronik AG Orsiro stent was performed via the REVERSE mechanism as we described in the report of the new intervention in the earlier section $(2.5 \times 18)$ (Figure 1).

Why did we use the modified flower petal stenting in the side branch and a strut in the main branch? The answer is that the strut contains drugs that damage endothelial cells in the branch wall and induce heparin release; so, it increases the risk of thrombosis. In modified flower petal stenting, the main stent that contains three layers (three multiplied drug dosage) is used in the main branch but in REVERSE modified flower petal stenting, and we used just one strut to decrease the risk of thrombosis in the main branch.

After the procedure and recovery, the patient was discharged from the hospital and followed for 19 months. The exercise stress test using the Bruce protocol was done to evaluate the success of the intervention (Figure 2). Figure 3 declares the ECG in stage 3 of the exercise stress test result.

\section{Discussion}

New effective techniques and methods have been developed and experienced in the past decades. Angioplasty is a common technique for patients who suffer from AMI that improves the outcome and prognosis of these patients (2). However, clinical studies demonstrated that the outcomes of PCI are not sufficient in patients with coronary bifurcation lesions. During the years after describing and evaluating the PCI, many stenting techniques have been designed and clinically experienced to improve the outcomes of PCI in AMI patients with bifurcation lesions (6). Accordingly, cullote, crush stenting, T-stenting, V-stenting, and kissing stent technique have been widely employed as two-stent methods for patients who suffer from complications resulting from CHD. Despite the successful clinical practices of these stenting techniques, there is strong evidence of late complications such as either restenosis or thrombosis due to stenting techniques using two stents (7). A study by Al Suwaidi et al. (8) showed that in these lesions, balloon angioplasty without stenting increases the risk of acute vessel closure; on the other hand, the implantation of bare metal stents (BMS) was associated with approximately $30 \%$ rate of Major adverse cardiac events (MACE) at one year of the study (8).

In the past decades, several techniques were introduced and experienced to overcome the limitations resulting from pPCI. The poor clinical outcomes due to pPCI are still reported from almost all countries (5). Several studies on coronary bifurcation lesions showed that the main vessel and the side branch need stent coverage. Recently, a new stenting technique named flower petal stenting was explained by Kinoshita and colleagues (9). Simultaneous multivessel epicardial coronary artery thrombosis (STEMI) generally leads to cardiogenic shock, as reported in a 42-year-old male patient presenting with acute anterior STEMI with triple coronary artery thrombosis. In this case, the physicians decided to perform PCI for the LAD; then, they used RCA thrombosis regressed with 24-hour tirofiban (glycoprotein IIb/IIIa receptor inhibitor) perfusion and used the flower petal technique. They reported thrombolysis in myocardial infarction (TIMI) flow grade III after stenting; their patient was discharged within five days after PCI and the treatment showed to be successful in this case (10).

During the past decade, the modified flower petal technique was introduced for stenting, particularly in cases with the presentation of bifurcation lesions (5). Modified flower petal is a very difficult but efficient angioplasty technique that has been electively performed until now. 

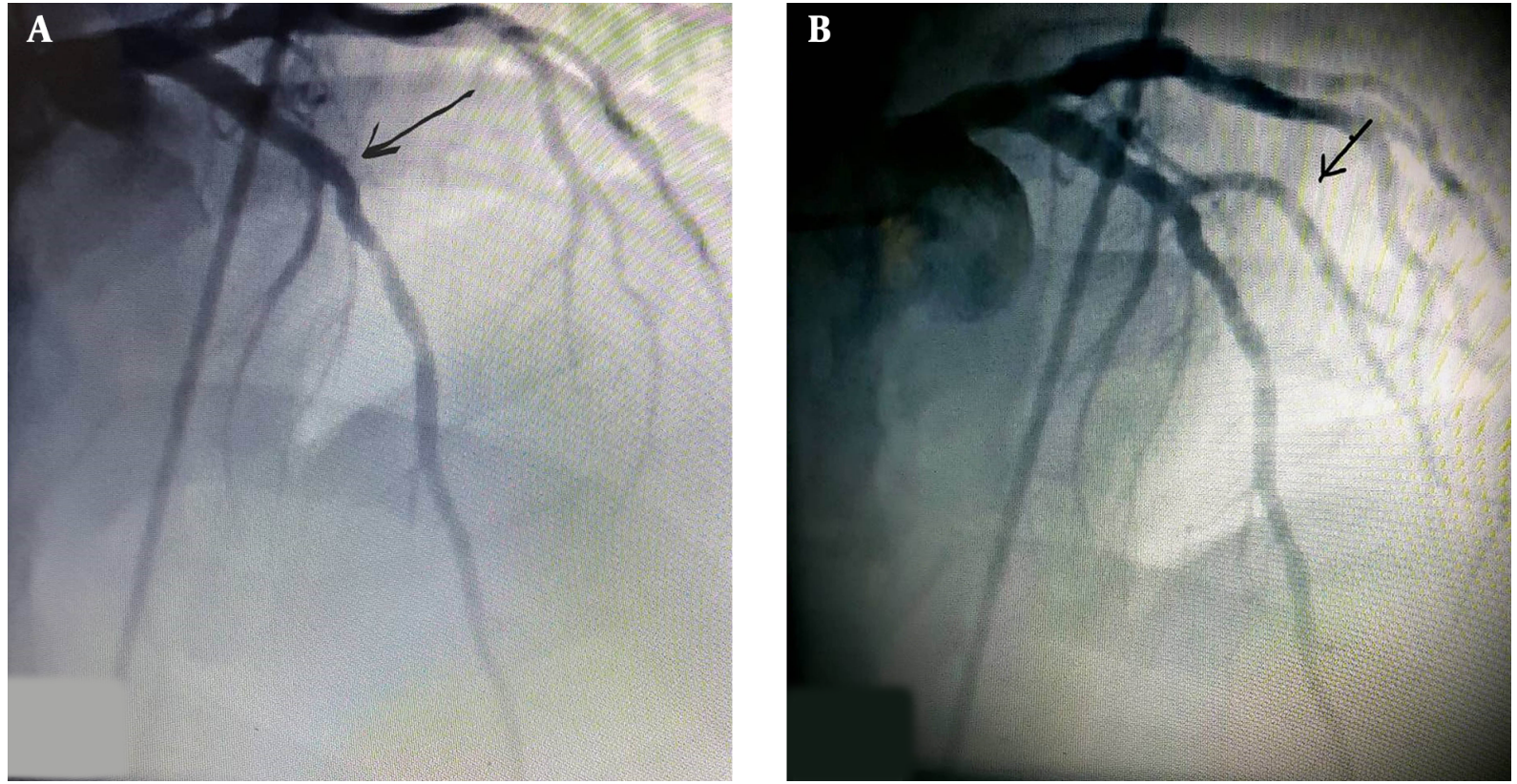

Figure 1. Angiography and primary percutaneous intervention indicating A, occlusion of the artery that B, was treated with the REVERSE modified flower petal stenting technique.
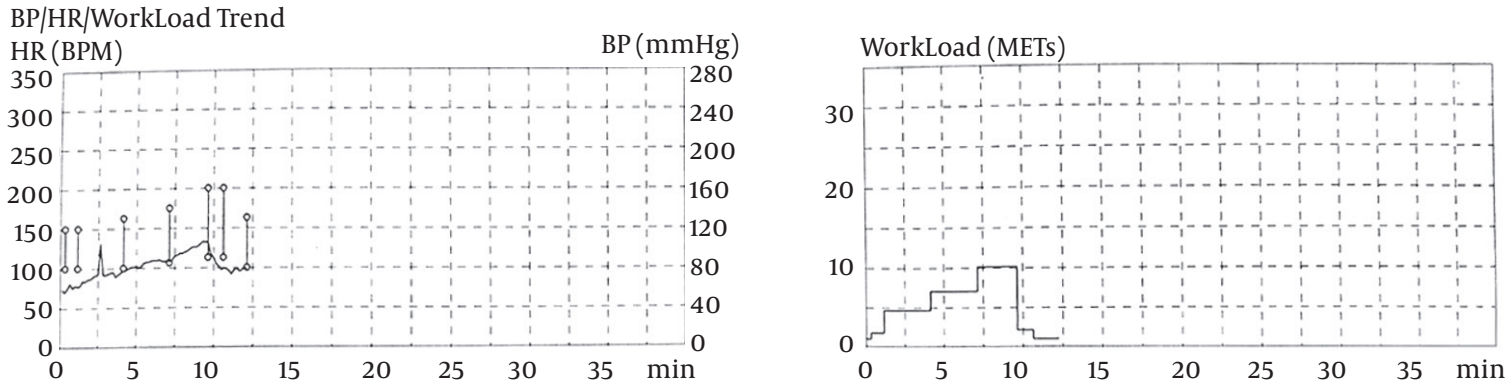

Summary Summarize

\begin{tabular}{|c|c|c|c|c|c|c|c|c|c|c|c|c|}
\hline \multirow{2}{*}{ No. } & \multirow{2}{*}{ Phase } & \multirow{2}{*}{$\begin{array}{l}\text { Stage Time } \\
\text { (min:sec) }\end{array}$} & \multirow{2}{*}{$\begin{array}{l}\text { Speed } \\
\text { (mph) }\end{array}$} & \multirow{2}{*}{$\begin{array}{c}\text { Grade } \\
(\%)\end{array}$} & \multirow{2}{*}{$\begin{array}{c}\mathrm{HR} \\
\text { (BPM) }\end{array}$} & \multicolumn{2}{|c|}{ BP } & \multirow{2}{*}{$\begin{array}{c}\text { NorkLoad } \\
\text { (METs) }\end{array}$} & \multicolumn{4}{|c|}{$\mathrm{ST}(\mathrm{mV})$} \\
\hline & & & & & & $\mathrm{mmHg}$ & $\mathrm{kPa}$ & & + & $\mathrm{L}$ & - & $\mathrm{L}$ \\
\hline 0 & Rest & $00: 23$ & 0.0 & 0.0 & 70 & $120 / 80$ & $16.0 / 10.7$ & 1.0 & 0.19 & I & -0.02 & I \\
\hline 1 & Warm up & 00:52 & 1.0 & 0.0 & 75 & $120 / 80$ & $16.0 / 10.7$ & 1.8 & 0.30 & V4 & -0.25 & $\mathrm{aVR}$ \\
\hline 2 & Stage 1 & 03:00 & 1.7 & 10.0 & 91 & $130 / 80$ & $17.3 / 10.7$ & 4.6 & 0.40 & V3 & -0.40 & V3 \\
\hline 3 & Stage 2 & 03:00 & 2.5 & 12.0 & 108 & $140 / 85$ & $18.7 / 11.3$ & 7.1 & 0.40 & V2 & -0.40 & V1 \\
\hline 4 & Stage 3 & 02.30 & 3.4 & 14.0 & 118 & $160 / 90$ & $21.3 / 12.0$ & 10.2 & 0.40 & I & -0.40 & I \\
\hline 5 & Recovery 1 & 01:00 & 1.5 & 0.0 & 97 & $160 / 90$ & $21.3 / 12.0$ & 2.1 & 0.40 & I & -0.40 & I \\
\hline 6 & Recovery 2 & $02: 28$ & 0.0 & 0.0 & 99 & $130 / 80$ & $17.3 / 10.7$ & 1.0 & 0.19 & V4 & -0.13 & aVL \\
\hline
\end{tabular}

Figure 2. Exercise stress test results of follow-up after 24 months 


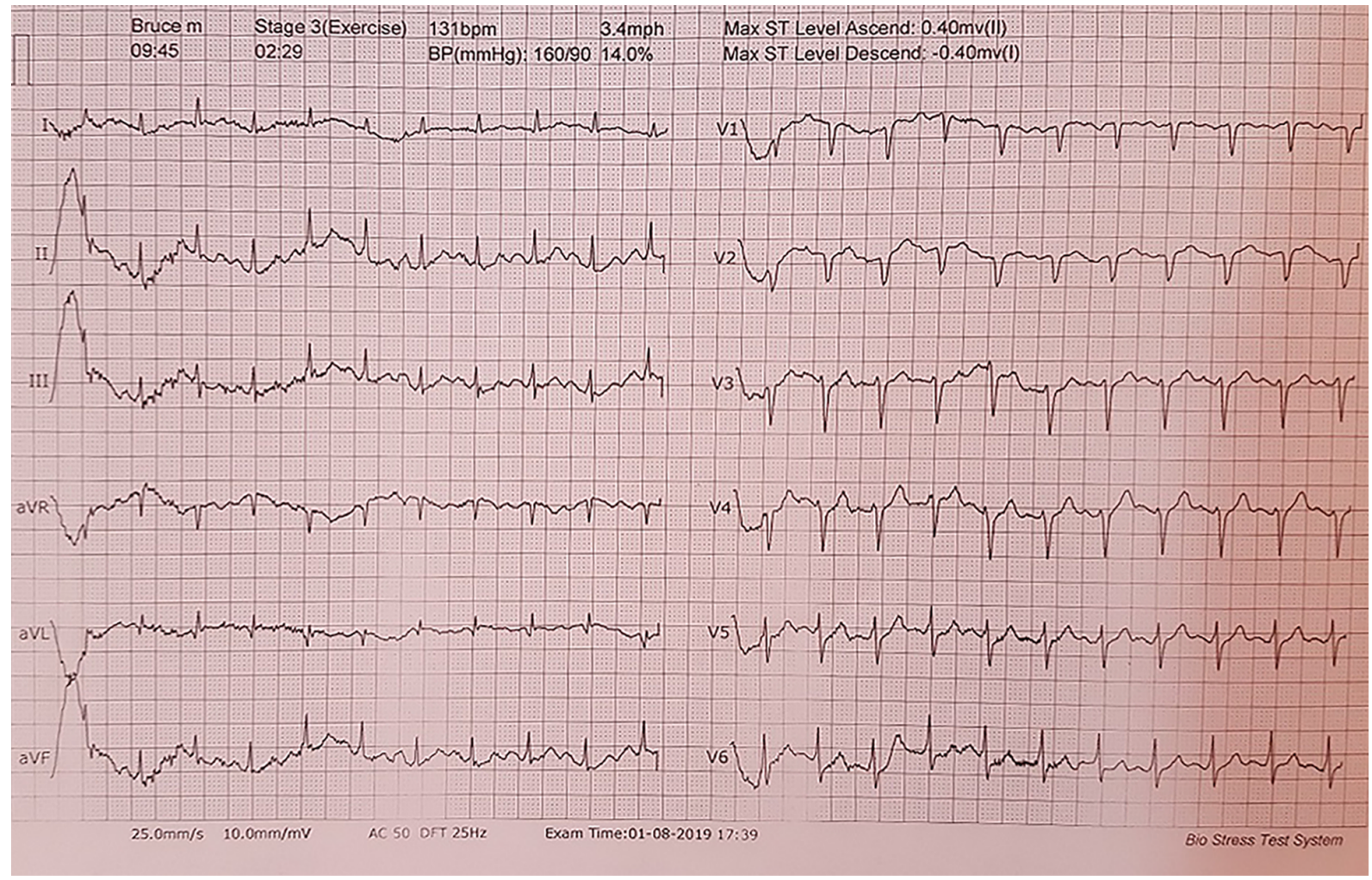

Figure 3. The ECG of stage 3 of the exercise stress test showing no change in cardiac function

However, reviewing the literature shows that there is not enough data about this technique all over the world. First, Kinoshita et al. (9) experienced this technique for patients with bifurcation lesions. However, they showed that there were no cases of restenosis in those undergoing the flower petal technique for stenting; this precious study by Kinoshita et al. (9) used the flower petal stenting technique worked by flaring the proximal side of the stent in the side branch out like a flower petal in an in vitro study in 33 patients; they observed that nine months of follow-up explained the complete coverage of bifurcation with minimal stent-layer overlapping and concluded that the flower petal stenting technique can be useful in the treatment of bifurcation lesions (9). In another study by Cayli et al. (5), the modified flower petal technique was used for patients who suffered from Medina type 1.1.1 coronary bifurcation lesions. Accordingly, they claimed $100 \%$ procedural success for patients who underwent this technique (5).

\subsection{Conclusions}

In comparison with the mentioned studies that experienced this technique electively, we used the flower petal technique for a case of AMI. Concerning the fact that the main goals during primary PCI in the current case were (1) decreasing the risk of late complications due to restenosis and thrombosis resulting from the employment of two stents and (2) increasing the chance of rescue of the coronary artery, we performed the modified flower petal technique. However, the 24-month follow-up of the patient showed satisfying outcomes and there was no evidence of restenosis. Therefore, concerning the promising results of the application of this technique in the case of AMI and the limitations of pPCI, explaining this case may help the interventionists facing AMI cases with bifurcation lesions.

\section{Acknowledgments}

We wish to thank all our colleagues and cooperators in Golestan Hospital, Ahvaz Jundishapur University of Medical Sciences, Ahvaz, Iran

\section{Footnotes}

Authors' Contribution: $\mathrm{HH}$ took care of the patient, performed the intervention, collected information, and re- 
vised the manuscript. ZDZ presented the case and wrote the manuscript.

Conflict of Interests: The authors declare that they have no conflict of interest.

Ethical Approval: This study was approved for publication by the Clinical Research and Ethics Committee of Ahvaz University of Medical Sciences. Ethics, consent, and permission were given for this study.

Funding/Support: There was no fund for the current study.

Informed Consent: Written informed consent was obtained from the patient for the publication of this case report and any accompanying images. A copy of written consent is available for review by the Editor-in-Chief of this journal.

\section{References}

1. Zijlstra F. Acute myocardial infarction: primary angioplasty. Heart. 2001;85(6):705-9. doi: 10.1136/heart.85.6.705. [PubMed: 11359760]. [PubMed Central: PMC1729777].

2. Khan KN, Khan MH, Rahman R, Rashid MA, Haque SZ, Zakia Z. Primary Angioplasty for the Treatment of Acute ST Elevated Myocardial Infarction: Single Centre Experience. Mymensingh Med J. 2017;26(2):351-5. [PubMed: 28588172].

3. Gibson CM. Primary angioplasty compared with thrombolysis: new issues in the era of glycoprotein IIb/IIIa inhibition and intracoronary stenting. Ann Intern Med. 1999;130(10):841-7. doi: 10.7326/0003-4819130-10-199905180-00019. [PubMed: 10366375].

4. Stankovic G, Lefevre T, Chieffo A, Hildick-Smith D, Lassen JF, Pan $\mathrm{M}$, et al. Consensus from the 7th European Bifurcation Club meeting. EuroIntervention. 2013;9(1):36-45. doi: 10.4244/EIJV9I1A7. [PubMed: 23552575].

5. Cayli M, Sahin DY, Elbasan Z, Gur M, Cagliyan CE, Kivrak A, et al. Modified flower petal technique: a new technique for the treatment of Medina type 1.1.1 coronary bifurcation lesions. JACC Cardiovasc Interv. 2013;6(5):516-22. doi: 10.1016/j.jcin.2012.12.130. [PubMed: 23702015].

6. Finch W, Lee MS. Percutaneous Coronary Intervention for Coronary Bifurcation Lesions. Rev Cardiovasc Med. 2017;18(2):59-66. [PubMed: 29038413].

7. Dash D. Recent perspective on coronary artery bifurcation interventions. Heart Asia. 2014;6(1):18-25. doi: 10.1136/heartasia-2013-010451. [PubMed: 27326157]. [PubMed Central: PMC4832703].

8. Al Suwaidi J, Yeh W, Cohen HA, Detre KM, Williams DO, Holmes DJ. Immediate and one-year outcome in patients with coronary bifurcation lesions in the modern era (NHLBI dynamic registry). Am J Cardiol. 2001;87(10):1139-44. doi: 10.1016/s0002-9149(01)01482-5. [PubMed: 11356386].

9. Kinoshita Y, Katoh O, Matsubara T, Ehara M, Nasu K, Habara M, et al. First clinical experience of "flower petal stenting": a novel technique for the treatment of coronary bifurcation lesions. JACC Cardiovasc Interv. 2010;3(1):58-65. doi: 10.1016/j.jcin.2009.09.016. [PubMed: 20129570].

10. Ermis E, Kahraman S, Ucar H, Ozyilmaz SO, Allahverdiyev S. Triple Coronary Artery Thrombosis Presenting as Acute Anterior STSegment Elevation Myocardial Infarction. Int J Angiol. 2018;27(4):2236. doi: 10.1055/s-0037-1608959. [PubMed: 30410294]. [PubMed Central: PMC6221801]. 